\title{
The Use of Polarization Filters to Detect the Edge of the Descemet's Stripping Automated Endothelial Keratoplasty(DSAEK) Graft
}

\author{
Takeshi Ide*, Sonia H. Yoo and Terrence P. O’Brien
}

Bascom Palmer Eye Institute, Miami, FL, USA

\begin{abstract}
Purpose: To report the technique of combining the two different polarization filters to detect the flap edge of the corneoscleral tissue before trephining the Descemet's stripping automated endothelial keratoplasty (DSAEK) tissue.

Methods: A human DSAEK donor tissue was prepared with mechanical microkeratome and the tissue on the cutting block was brought under the microscope. The liner, circular, or the combination of these two polarization filters was placed between the tissue and the microscope. The tissue images were taken with digital camera under either of 3 settings.

Results: The combination of circular and linear polarization filters enabled us to recognize the edge of the flap more easily than others.

Conclusion: This simple system with polarization filters was effective in clear visualization of the flap edge during DSAEK tissue preparation. These features may significantly enhance safety of various surgical procedures, in addition to DSAEK tissue preparation.
\end{abstract}

Keywords: DSAEK, polarization filter, edge, flap, circular, linear.

\section{INTRODUCTION}

Recently, numbers of attempts have been made to selectively replace the endothelial cell layer of the cornea in order to reduce the complications of penetrating keratoplasty (PKP) $[1,2]$. Descemet's stripping automated endothelial keratoplasty (DSAEK) has recently become the most popular selective endothelial keratoplasty(EK) method, mainly because of the shorter surgery time, the subsequent rapid visual rehabilitation and the less induced astigmatism [3-7]. The DSAEK procedure selectively replaces diseased corneal endothelium with donor posterior stroma and endothelium through a corneal/corneoscleral incision.

In spite of its popularity, several steps can potentially limit the success of DSAEK. Trephining the DSAEK donor tissue from the microkeratome prepared corneoscleral tissue is among these limiting steps, though they appear to look easy. Whether tissue is prepared by surgeons, eye banks or tissue distributing companies, surgeons have to punch out the tissue just before the tissue insertion into the patients' anterior chamber (AC). The microkeratome cut is sometimes not centered on the corneal geometrical center. And, the stromal side is sometimes not stained with marking ink to avoid the endothelial death [8]. In addition, in trephining the tissue, the endothelial side of the donor tissue is often covered with storage media, balanced salt solution (BSS), ophthalmic viscoelastic device (OVD) with endothelial side up on the cutting block. Due to these factors and light reflection, the edge of the flap is sometimes hard to be recognized. In this investigation, we test the easy way to detect the edge of the flap using commercially available polarization filters.

*Address correspondence to this author at the Bascom Palmer Eye Institute, 900 NW 17th Street, Miami, Florida 33136, USA; Tel: +1-305 326 6322; Fax: +1-305 326 6337; E-mail: teyede@hotmail.com

\section{MATERIALS AND METHODS}

A human cornea was obtained from the Eye Bank, which was unacceptable for clinical transplantation. The epithelium was scraped off from the corneas followed by microkeratome-assisted excision of the anterior lamellar corneal tissue with a Moria artificial anterior chamber using a 300-micron $\mathrm{CB}$ head (Moria Systems, Doylestown, PA). The anterior lamellar corneal tissue was placed back onto the stromal bed [9], and the tissue was put back into Optisol ${ }^{\circledR}$ GS solution (Bausch \& Lomb Surgical, Irvine, CA) at $4^{\circ} \mathrm{C}$ until use.

Before trephining the DSAEK tissue from the corneoscleral rim, either the linear polarizer filter (PL), circular polarizer (CPL) or the combination of these two filters was placed between the tissue on the cutting block and the microscope and digital photographs were taken.

\section{RESULTS}

Photographs for each condition are shown in Fig. (1). The PL or CPL alone enhanced the image quality under the microscope. The combination of CPL and PL, however, enabled us to recognize the edge of the flap edge much more easily by rotating the relative angle between the PL and CPL (Fig. 1).

\section{DISCUSSION}

The DSAEK procedure is becoming more popular and has many theoretical advantages over conventional PKP for patients with diseased or traumatized endothelium without anterior corneal scarring; the shorter surgery time, the faster visual rehabilitation and less irregular astigmatism than penetrating keratoplasty (PKP). However, DSAEK is not free from problems. One of the issues is the difficulty in the trephination of the tissue inside the flap bed, especially for small diameter microkeratome-assisted flap globes. This is 

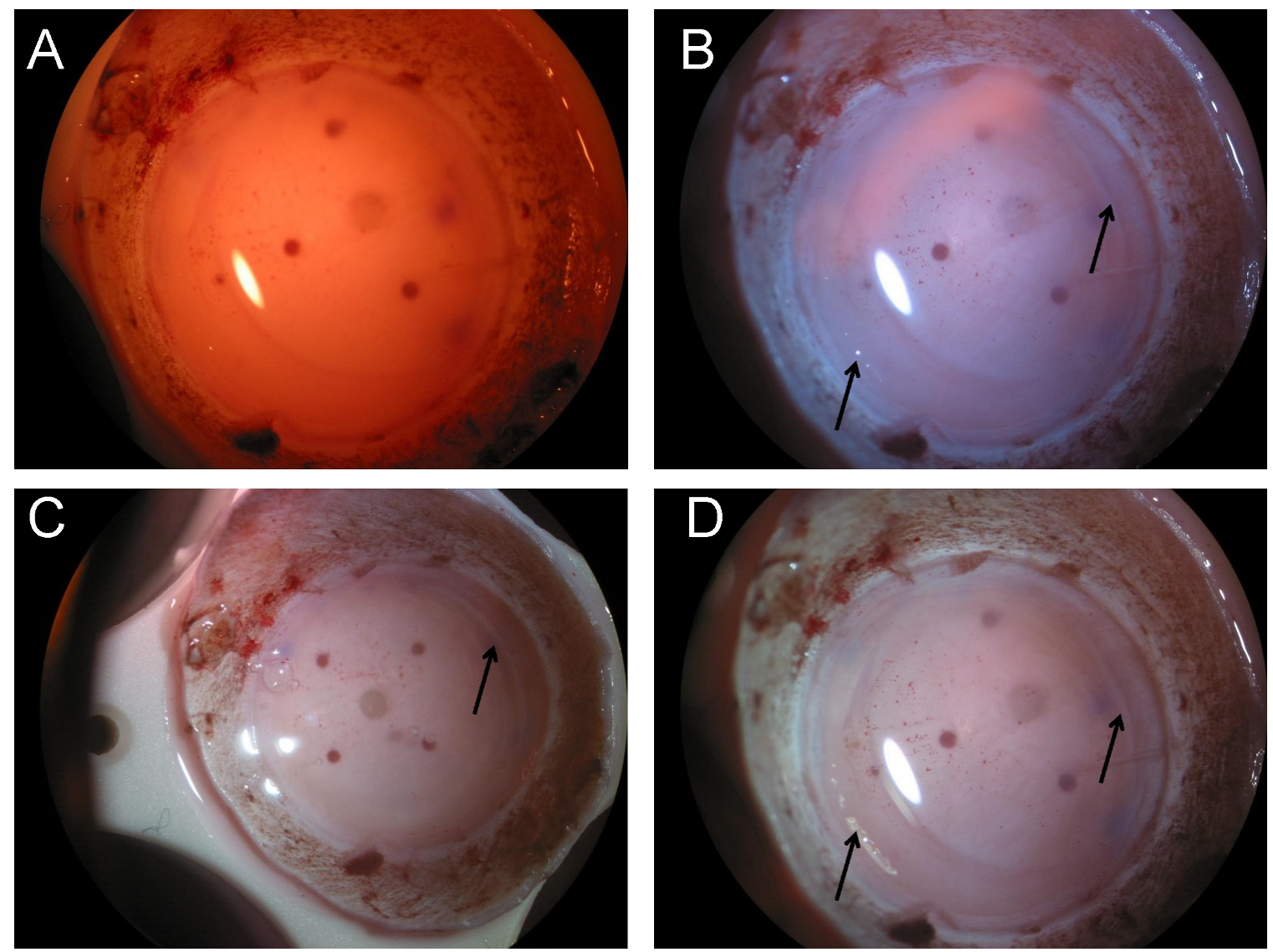

Fig. (1). Images of DSAEK tissue filtered by polarizer. The edge of the stromal side flap edge is observed most clearly in $\mathbf{D}$ (combination of the two filters) followed by $\mathbf{B}$ (circular polarizer) and $\mathbf{C}$ (linear polarizer). We could not detect any edge in $\mathbf{A}$ (control without filter) in this tissue.

mainly due to the invisibility of the flap edge of the corneoscleral rims. To facilitate the visualization of the tissue edge, we usually mark the cornea outside of the tissue and adjust the light settings or tilt the tissue on the cutting block. However, difficulty still remains to define the edge of the DSAEK tissue, as the staining is diffused during the storage period.

Slitlamp users are aware that while examining the cornea, a fully opened slit gives an overall view of the cornea but fine details of corneal structures and opacities are often obscured. A complete corneal examination requires a selection of lighting techniques to appreciate the delicate detail of corneal structures. A fine slit viewed stereoscopically moving across the cornea allows the user to appreciate corneal translucence and topography. However, photographic documentation is limited by a single, static photograph. When direct, focal, wide-slit illumination is used to image the cornea, the high reflectivity of the moist corneal surface impairs visualization of the surface and underlying structures.

Likewise, in DSAEK tissue trephination, we have to observe the flap edge underlying the corneal endothelium, Descemet's membrane and stroma fibers with bright broad illumination. An analogy is the sun reflecting from a window obscuring the room beyond. Conventionally, photographers have used polarizing filters to minimize reflections, glare, and flare from highly reflective surfaces. Barry et al. utilized polarization filters to reduce the unwanted light reflection to image the LASIK flap edge more clearly $[10,11]$. In this investigation, we also tried this antireflection strategy with polarization filter. We utilized two kinds of commercially available polarizer: circular (CPL) and linear (PL).

Each alone can control the light reflex and worked moderately but we could not detect the edge sufficiently. Then, we tried the combination of these two filters to control also the light wavelength (colors) in addition to the direction of the light (Fig. 2), because white light source from the microscope has prism effect and can cause image obscureness. Described in the results, this double polarized filtration effectively visualized the edge of the stromal edge of the flap more clearly than the view without at specific range of the angle between CPL and PL.

This cross-polarized filtration partially explains enhanced corneal viewing. A second, less-well-understood phenomenon is that of birefringence. Birefringence is the ability of a material to change the plane of polarization of the incident light. The cornea is said to be birefringent [12]. This effect has been described as a result of the stromal fibers crossing each other at near right angles, inducing this birefringent effect [12-14].

Though we are currently unsure which effect plays a major role when using polarizing filters to improve corneal images and we did randomly rotated the filter to find the optimal condition in this investigation, this simple system with easily and reasonably available polarization filters did work 

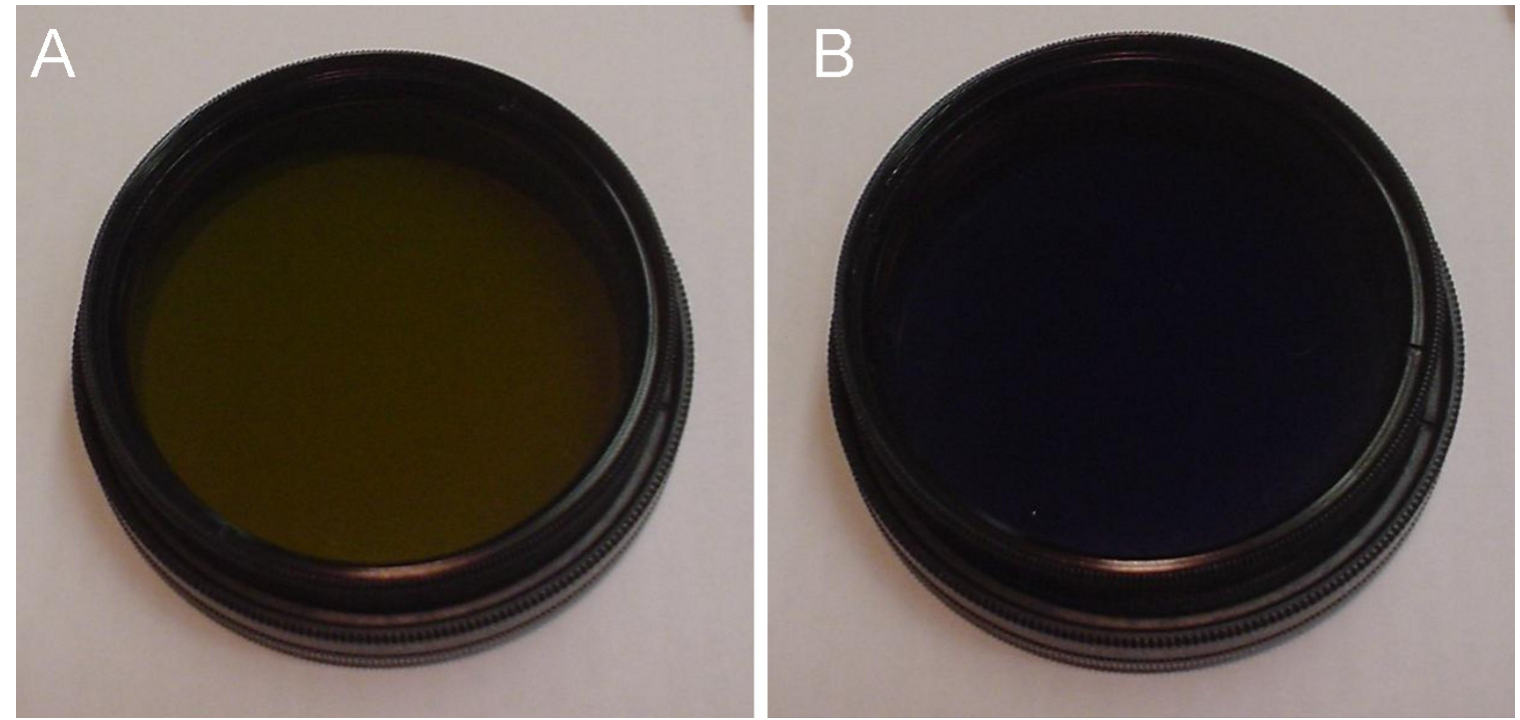

Fig. (2). Combined circular and linear filter. By rotating and changing the relative angle between CPL and PL, the color change of the filter can be observed. (A, B).

for visualizing the flap edge in the DSAEK tissue preparation by controlling the rate of transparency and wave length of light. In DSAEK surgery, consistent tissue quality is important, because unexpected decentered trephination can yield partially thickened graft, resulting in more endothelial damage during the delivering the tissue into the anterior chamber.

Therefore, these features of polarization filters may significantly enhance the ease and the safety of various surgical procedures from anterior to posterior segment surgeries, in addition to DSAEK tissue preparation.

$$
\begin{aligned}
& \text { ABBREVIATIONS } \\
& \text { PKP = Penetrating keratoplasty } \\
& \text { DSAEK = Descemet's Stripping Automated Endothelial } \\
& \text { EK }=\text { Endothelial keratoplasty } \\
& \mathrm{AC}=\text { Anterior chamber } \\
& \text { BSS }=\text { Balanced salt solution } \\
& \text { OVD = Ophthalmic viscoelastic device } \\
& \text { LASIK = Laser in situ keratomileusis } \\
& \mathrm{PL}=\text { Linear polarizer filter } \\
& \mathrm{CPL}=\text { Circular polarizer }
\end{aligned}
$$

\section{REFERENCES}

[1] Culbertson WW. Descemet stripping endothelial keratoplasty. Int Ophthalmol Clin 2006; 46: 155-68.
[2] Terry MA. Endothelial keratoplasty: history, current state, and future directions. Cornea 2006; 25: 873-8.

[3] Fang JP, Hamill MB. Descemet's stripping endothelial keratoplasty under topical anesthesia. J Refract Surg 2007; 33: 187-8.

[4] Koenig SB, Covert DJ. Early results of small-incision Descemet's stripping and automated endothelial keratoplasty. Ophthalmology 2007; 114: 221-6.

[5] Price FW Jr, Price MO. Descemet's stripping with endothelial keratoplasty in 200 eyes: early challenges and techniques to enhance donor adherence. J Refract Surg 2006; 32: 411-8.

[6] Terry MA, Hoar KL, Wall J, et al. Histology of dislocations in endothelial keratoplasty (DSAEK and DLEK): a laboratory-based, surgical solution to dislocation in 100 consecutive DSAEK cases. Cornea 2006; 25: 926-32.

[7] Price MO, Price FW Jr. Descemet's stripping with endothelial keratoplasty: comparative outcomes with microkeratome-dissected and manually dissected donor tissue. Ophthalmology 2006; 113 : 1936-42.

[8] Ide T, Yoo SH, Kymionis GD, Perez VL, Goldman JM, O'Brien TP. Descemet-stripping automated endothelial keratoplasty (DSAEK): effect of nontoxic gentian violet marking pen on DSAEK donor tissue viability by using vital dye assay. Cornea 2008; 27: 562-4.

[9] Ide T, Yoo SH, Kymionis GD, Goldman JM, Perez VL, O'Brien TP. Descemet-stripping automated endothelial keratoplasty: effect of anterior lamellar corneal tissue-on/-off storage condition on Descemet-stripping automated endothelial keratoplasty donor tissue. Cornea 2008; 27: 754-7.

[10] Peli E. Ophthalmic applications of circular polarizers. J Am Optom Assoc 1986; 57: 298-302.

[11] Barry CJ, Crawford GJ. Dislocated laser in situ keratomileusis flap visualized by cross-polarized filtration. J Cataract Refract Surg 2002; 28: 1478-80

[12] Mountford J. Polarized biomicroscopy. Int Contact Lens Clin 1982; 9: 373-84.

[13] Mishima S. The biomicroscopy of the human eye using polarized light; findings in normal cornea. Jpn J Ophthlmol 1958; 2: 182-92.

[14] Stanworth A, Naylor EJ. The polarization optics of the isolated cornea. Br J Ophthalmol 1950; 34: 201-11. $\mathrm{nc} / 3.0 /$ ) which permits unrestricted, non-commercial use, distribution and reproduction in any medium, provided the work is properly cited. 